\title{
Solution of the Puff Model for Pollutant Dispersion in the Atmospheric Boundary Layer by an Analytical Approach
}

\author{
Everson da Silva, Marco Tullio Vilhena, \\ Programa de Pós-Graduação em Engenharia Mecânica, PROMEC, UFRGS \\ 90050-170, Porto Alegre, RS \\ E-mail: eversonjg@gmail.com, $\quad$ vilhena@pq.cnpq.br \\ Tiziano Tirabassi, \\ ISAC - Institute ISAC of CNR \\ Bologna, Italy \\ E-mail: t.tirabassi@isac.cnr.it \\ Daniela Buske \\ Programa de Pós-Graduação em Modelagem Matemática, PPGMMat, UFPel \\ 96160-000, Pelotas, RS \\ E-mail: danielabuske@pq.cnpq.br
}

\begin{abstract}
The objective of this work is to present an analytical approach for the solution of the advection-diffusion equation that describes a puff model. This model simulate the behavior of a pollutant for an instantaneous emission in non homogeneous and non stationary meteorological conditions. The GILTT method (Generalized Integral Laplace Transform Technique) was used to solve the problem. With the model presented in this work we aim to refine an earlier one, which assumes a homogeneous longitudinal diffusion, using a Gaussian in this direction. For this purpose, in the present work, we solve a transient three-dimensional problem taking into account the diffusion coefficients in all directions. For comparison and validation of the proposed model we used data from the experiment of Copenhagen and the results obtained with the Gaussian model.
\end{abstract}

Keywords: Air pollution, air pollution modeling, puff models, advection-diffusion equation, GILTT method.

\section{Introduction}

The management and safeguard of air quality presupposes knowledge of the state of the environment. Such knowledge involves both cognitive and interpretative aspects. The processes governing the transport and diffusion of pollutants are numerous, and of such complexity that it would be impossible to describe them without the use of mathematical models. Such models therefore constitute an indispensable technical instrument of the air quality management. There exist innumerable, sometimes very diverse, mathematical models of atmospheric pollutant diffusion that may be utilized for the aforementioned purposes. In fact, the phenomenon of turbulent diffusion in the atmosphere has no single formulation, in the sense that any one available approach can explain all the observed phenomena.

In this realm, puff models are practical operative models to simulate the pollutant dispersion in non-homogeneous and non-stationary atmospheric scenarios due a source emission. The source emission is discretized in such successive set of puffs that each puff moves toward the area of calculus, driven by the three-dimensional wind profile which varies with time. 
Puff models are quite suitable to handle the pollutant dispersion simulation in atmosphere over complex terrain. Their practical and theoretical simplicity, besides its flexibility are the reasons for the great success of this kind of model for pollutant dispersion. In the first puff models appearing in literature, the pollutant diffusion in the puffs is modeled by the Gaussian formulation. However, in the last years, the literature manifests some advances for such models. In fact, for instance, [9] proposed an approximate solution for describing the dispersion of a cloud of passive contaminants released from an instantaneous source near the ground. In [9] puff velocity and rate equations for the standard deviations of the concentration distribution and for the skewness are derived. [7] improved the puff model for a continuously emitting source by the superposition of a series of the cited cloud. [8] presented a puff model using the truncated Gram-Charlier expansion of the concentration field and the finite set of equations for the corresponding moments for solving the advection-diffusion equation.

Recently, [5] discussed two puff models with the main feature that the horizontal dispersion is expressed by a Gaussian formula, meanwhile the vertical puff shape is non-Gaussian and it is evaluated by two different techniques that allow to obtain an analytical solution of the onedimensional advection-diffusion equation: the ADMM (Advection Diffusion Multilayer Method) and GILTT (Generalized Integral Laplace Transform Technique) techniques. The first one is a model based on a discretization of the Atmospheric Boundary Layer (ABL) and the solution is given in integral form. The main feature of this method relies on the following steps: stepwise approximation of the eddy diffusivity and wind speed, double Laplace transform application to the advection-diffusion equation in the $\mathrm{x}$ and $\mathrm{t}$ variable, semi-analytical solution of the set of linear ordinary equation resulting for the double Laplace transform application and construction of the pollutant concentration by the Laplace transform inversion using the Gaussian quadrature scheme (semi-analytical due to the numeric inversion). For more details see the work [4]. The second one skips the multilayer discretization of the height $\mathrm{z}$ appearing in the ADMM approach and is given in series form. Briefly speaking, the idea of GILTT approach comprehends the steps: expansion of the concentration in series of eigenfunctions attained from an auxiliary problem, replacing this equation in the advection-diffusion equation and taking moments, one comes out with a matrix ordinary differential equation that is then solved analytically by the Laplace Transform technique. For more information see the work [3]. Both models, described above, accept general profiles for eddy diffusivity coefficients, as well as the theoretical profiles proposed in the scientific literature, such as the vertical profiles of eddy diffusion coefficients predicted by the Similarity Theory.

In this work, a non-Gaussian puff model, whose dispersion of the pollutant inside the puff in the three directions $(\mathrm{x}, \mathrm{y}, \mathrm{z})$ are written as a product of one-dimensional GILTT analytical solutions, is presented. At this point, is important recall that the GILTT solution is derived for an eddy-diffusivity depending on the vertical direction. Therefore this formulation is quite appropriate to simulate pollutant dispersion in non-homogeneous atmosphere. Further, by analytical the authors mean that no approximation is done along the solution derivation [3]. Finally, numerical simulations for the concentration at ground and statistical comparisons against experimental data are reported.

\section{The puff model}

Puff models are operative models that allow discretizing the transport and dispersion over time (through a set of discrete puffs) and separate transport and dispersion. In fact, the transport is simulated through the trajectory of the different puffs, while the dispersion is represented by the expansions of the puffs. That is, each puff is carried in accordance with the trajectory from its centre, which is determined for velocity vector of the local wind, while it is enlarged in the time by means of the dispersion coefficients describing the atmospheric turbulence.

Puff models assume that each emission of pollutants in a time interval $\Delta t$ releases into the atmosphere a mass of pollutants $\Delta M=Q \Delta t$, where $\mathrm{Q}$ is the emission rate, which is variable in 
time. A puff release scenario assumes that the release time and sampling times are very short compared to the travel time from the source to the receptor. For this reason puff models can be used to simulate diffusion in calm wind conditions too.

Each puff contains the mass $\Delta M$ and it is transported by the wind, which may vary in space and time. In a puff model the wind velocity influences the calculation of the concentration only in the density of puffs in the region of diffusion (the lower the wind velocity, the closer the puffs emitted by a source).

Each puff has his trajectory described by its center, which is defined as follows:

$$
x_{0}=\bar{u} \Delta t \quad y_{0}=\bar{v} \Delta t \quad z_{0}=\bar{w} \Delta t
$$

Consequently the total concentration of a pollutant in a given point in space is given by the sum of all puffs issued, namely:

$$
C_{T}(x, y, z, t)=\sum_{\text {puff }=1}^{\text {total of puffs }} \triangle M_{\text {puff }}\left\{\int_{t=0}^{\infty} c_{\text {puff }}(x, y, z, t) H\left(t-t_{0}\right) d t\right\}
$$

where $\mathrm{H}$ is the Heaviside function and:

$$
c_{\text {puff }}(x, y, z, t)=c_{1}(x, t) c_{2}(y, t) c_{3}(z, t)
$$

As previously mentioned the three functions $c_{1}, c_{2}$ and $c_{3}$ for the Gaussian model reads like:

$$
\begin{gathered}
c_{1}=\frac{1}{\sqrt{2 \pi} \sigma_{x}} \exp \left[-\frac{1}{2}\left(\frac{x-x_{0}}{\sigma_{x}}\right)^{2}\right] ; c_{2}=\frac{1}{\sqrt{2 \pi} \sigma_{y}} \exp \left[-\frac{1}{2}\left(\frac{y-y_{0}}{\sigma_{y}}\right)^{2}\right] \\
c_{3}=\frac{1}{\sqrt{2 \pi} \sigma_{z}} \exp \left[-\frac{1}{2}\left(\frac{z-z_{0}}{\sigma_{z}}\right)^{2}\right]
\end{gathered}
$$

where $\sigma_{x}, \sigma_{y}$ and $\sigma_{z}$ are empirical diffusion coefficients.

On the other hand, in the work of [5] the functions $c_{1}$ and $c_{2}$ are Gaussian, but $c_{3}$ is the solution of the vertical one-dimensional advection-diffusion equation obtained with the GILTT method. Here, one improves the puff model considering that all the three functions $\left(c_{1}, c_{2}, c_{3}\right)$ are GILTT solutions of following diffusion problems. Considering that $c_{1}(x, t)$ is solution of the following problem:

$$
\frac{\partial c_{1}(x, t)}{\partial t}=\frac{\partial}{\partial x}\left(K_{x} \frac{\partial c_{1}(x, t)}{\partial x}\right)+Q \delta\left(x-x_{0}\right) \delta\left(t-t_{0}\right)
$$

with initial and boundary conditions:

$$
\begin{aligned}
& K_{x} \frac{\partial c_{1}}{\partial x}=0 \quad \text { at } \quad x=0, \quad x=-L_{x} ; L_{x} \\
& c_{1}(x, t)=0 \quad \text { at } \quad t=0
\end{aligned}
$$

Further, $c_{2}(y, t)$ satisfy the problem:

$$
\frac{\partial c_{2}(y, t)}{\partial t}=\frac{\partial}{\partial y}\left(K_{y} \frac{\partial c_{2}(y, t)}{\partial y}\right)+Q \delta\left(y-y_{0}\right) \delta\left(t-t_{0}\right)
$$

with initial and boundary conditions:

$$
K_{y} \frac{\partial c_{2}}{\partial y}=0 \quad \text { at } \quad y=0, \quad y=-L_{y} ; L_{y}
$$




$$
c_{2}(y, t)=0 \quad \text { at } \quad t=0
$$

And, finally, $c_{3}(z, t)$ is the solution of the equation:

$$
\frac{\partial c_{3}(z, t)}{\partial t}=\frac{\partial}{\partial z}\left(K_{z} \frac{\partial c_{3}(z, t)}{\partial z}\right)+Q \delta\left(z-H_{s}\right) \delta\left(t-t_{0}\right)
$$

with initial and boundary conditions:

$$
\begin{gathered}
K_{z} \frac{\partial c_{3}}{\partial z}=0 \quad \text { at } \quad z=0, \quad z=z_{i} \\
c_{3}(z, t)=0 \quad \text { at } \quad t=0
\end{gathered}
$$

Here, one assumes that the solution is symmetric in the $x$ and $y$ directions. $c_{i}$ denotes the mean concentration of a passive contaminant $\left(\mathrm{g} / \mathrm{m}^{3}\right), K_{x}, K_{y}$ and $K_{z}$ are the eddy diffusivities $\left(\mathrm{m}^{2} / \mathrm{s}\right)$ in the $x, y$ and $z$ directions, respectively, $Q$ is the emission rate $(\mathrm{g} / \mathrm{s}), h$ the height of the atmospheric boundary layer $(m), H_{s}$ the height of the source $(m)$ and $\delta$ represents the Dirac delta function.

\section{The one-dimensional GILTT solution}

The three equations (6,9 and 12) have to be solved. In the case of Eq. (12), the equation is rewritten like:

$$
\frac{\partial c(z, t)}{\partial t}=K_{z}^{\prime} \frac{\partial c(z, t)}{\partial z}+K_{z} \frac{\partial^{2} c(z, t)}{\partial z^{2}}+Q \delta\left(z-H_{s}\right) \delta\left(t-t_{0}\right)
$$

According [3] the solution of problem (15) is written like:

$$
c(z, t)=\sum_{n=0}^{N} c_{n}(t) \zeta_{n}(z)
$$

where $\zeta_{n}(z)=\cos \left(\lambda_{n} z\right)$ and $\lambda_{n}=n \pi / z_{i}$ are respectively the eigenfunctions and eigenvalues, and $c_{n}(t)$ is the solution of the transformed problem.

Replacing the equation (16) in equation (15) and taking moments the problem can be rewritten as a first order linear matrix equation like:

$$
Y^{\prime}(t)+F . Y(t)=\eta \delta\left(t-t_{0}\right)
$$

for $t>0$, where the matrix $\mathrm{F}$ is defined as $F=A^{-1} B$ with entries of matrices $\mathrm{A}$ and $\mathrm{B}$ defined as:

$$
\begin{gathered}
A=(a)_{n, m}=\int_{0}^{z_{i}} \zeta_{n}(z) \zeta_{m}(z) d z \\
B=(b)_{n, m}=\lambda_{n}^{2} \int_{0}^{z_{i}} K_{z} \zeta_{n}(z) \zeta_{m}(z) d z-\int_{0}^{z_{i}} K_{z}^{\prime} \zeta_{n}^{\prime}(z) \zeta_{m}(z) d z
\end{gathered}
$$

and $\eta$ is the vector $\eta=A^{-1} Q \zeta_{m}\left(H_{s}\right)$. For the initial condition, the procedure is analogous and after the substitutions due and integrations, the result is $Y(0)=c(z, 0)=0$. In this work the transformed problem represented by equation (17) is solved by the Laplace Transform technique and diagonalization.

For the Eq. (6) and Eq. (9) the procedure is the same. The state-of-art the GILTT method can be found in [3]. 


\section{Experimental data}

The performances of the present models were evaluated against Copenhagen experimental data set [2]. In the Copenhagen experiment the tracer SF6 was released without buoyancy from a tower at a height of $115 \mathrm{~m}$, and collected at the ground level positions at a maximum of three crosswind arcs of tracer sampling units. The sampling units were positioned, at the ground level, 2-6 $\mathrm{km}$ from the point of release. The site was mainly residential with a roughness length of 0,6 $\mathrm{m}$. The meteorological conditions during the dispersion experiments ranged from moderately unstable to convective. The values of the maximum crosswind concentrations normalized with the tracer release rate from [2] were used. Generally, the distributed data set contains hourly mean values of concentrations and meteorological data. However, in this model validation, meteorology data with a greater time resolution kindly made available by Gryning and described in [8] were utilized. In particular, 10 minutes averaged values for meteorological data were used.

The puffs considered here are emitted in time intervals $\Delta t_{1}=600 \mathrm{~s}$ and the calculation of the concentration of pollutants is made with a time resolution $\Delta t_{2}=60 \mathrm{~s}$.

\section{Atmospheric boundary layer parameterization}

In order to evaluate the performance of the puff model (called here as GILTT-puff) against experimental ground-level concentration a boundary layer parameterization have to be introduced.

In this work, for the vertical eddy diffusivity coefficient were adopted the formulations proposed by [6] and written as:

$$
K_{z}=\kappa w_{*} z\left(1-\frac{z}{z_{i}}\right)
$$

where $\kappa$ is the von-Kármán constant, $w_{*}$ is the convective velocity, $z$ is the vertical coordinate and $z_{i}$ is the boundary layer height. While for the lateral ones was used the formulation:

$$
K_{\alpha}=0.1 w_{*} z_{i}
$$

where $\alpha$ represents $x$ and $y$ directions respectively.

The wind speed profile has been parameterized following the similarity theory of MoninObukhov and OML model:

$$
\begin{gathered}
U=\frac{u_{*}}{0.4}\left[\ln \left(\frac{z}{z_{0}}\right)-\psi_{m}\left(\frac{z}{L}\right)+\psi_{m}\left(\frac{z_{0}}{L}\right)\right] \quad \text { if } \quad z \leq z_{b} \\
U=U\left(z_{b}\right) \quad \text { if } \quad z \leq z_{b}
\end{gathered}
$$

where $z_{b}=\min \left[|L|, 0.1 z_{i}\right]$, and $\psi$ is a stability function given by:

$$
\psi_{m}\left(\frac{z}{L}\right)=\ln \left(\frac{1+\gamma^{2}}{2}\right)+\ln \left(\frac{1+\gamma}{2}\right)^{2}-2 \arctan \gamma+\frac{\pi}{2},
$$

with $\gamma=\left(1-15 \frac{z}{L}\right)^{1 / 4}$.

\section{Model evaluation against experiment data.}

The authors applied the model using the Copenhagen experimental data set presented above. The model evaluation has to be considered preliminary because the data set considers neutralconvective ABL only.

Figure 1 shows the observed and predicted scatter diagram of predicted and measured data and the dotted line indicate the data that are in a factor two (that is, the values of predicted data are between half and twice as experimental ones). This figure, with the $67 \%$ of the data 
in a factor two, shows that a reasonable agreement is obtained between experimental data and the puff model.

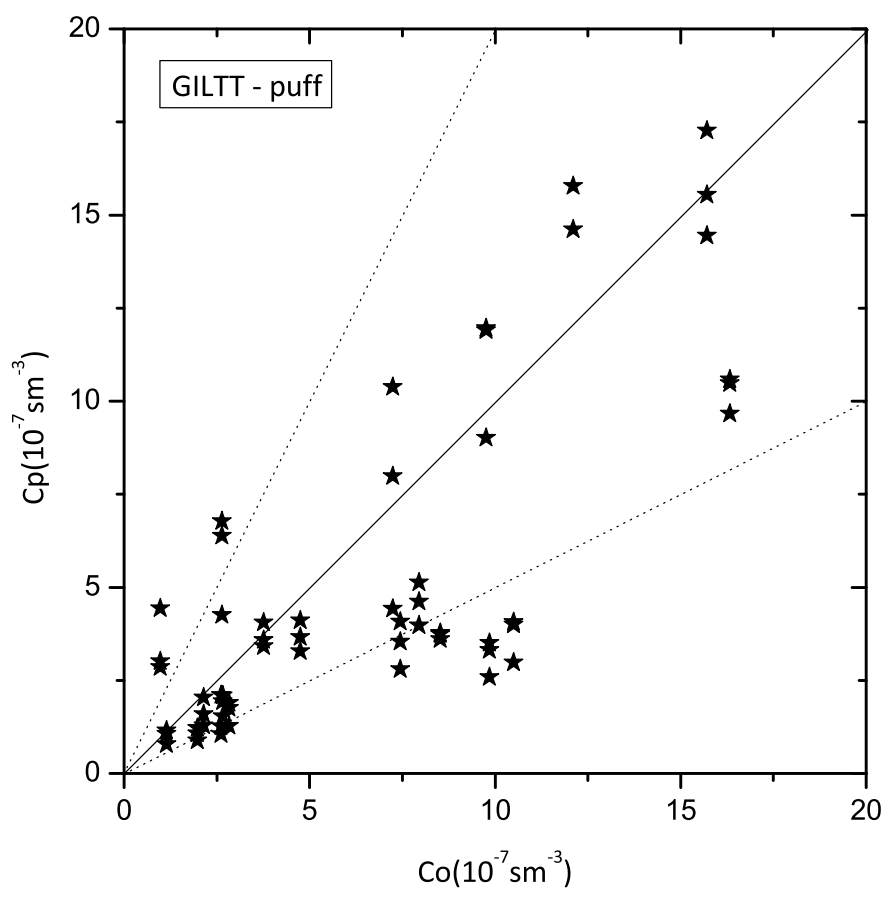

Figure 1: Observed and predicted scatter diagram of ground-level maximum crosswind concentration using the GILTT-puff approach. Lines indicate a factor of two.

Table 1 presents some popular performance measurements, obtained using the statistical evaluation procedure defined in [1]:

NMSE (normalized mean square error) $=\overline{\left(C_{o}-C_{p}\right)^{2}} / \overline{C_{o}} \overline{C_{p}}$

COR (correlation coefficient) $=\overline{\left(C_{o}-\overline{C_{o}}\right)\left(C_{p}-\overline{C_{p}}\right)} / \sigma_{o} \sigma_{p}$

FA2 (factor of 2$)=C_{p} / C_{o} \epsilon[0.5,2]$

$\mathrm{FB}($ fractional bias $)=\left(\overline{C_{o}}-\overline{C_{p}}\right) /\left(0.5\left(\overline{C_{o}}+\overline{C_{p}}\right)\right)$

FS (fractional standard deviation $)=2\left(\sigma_{o}-\sigma_{p}\right) /\left(\sigma_{o}+\sigma_{p}\right)$

where subscripts $\mathbf{o}$ and $\mathbf{p}$ refer to observed and predicted quantities, respectively, $\sigma$ is the standard deviation, $\mathbf{C}$ the concentration and the over bar indicates an averaged value. The statistical index FB says if the predicted quantities underestimate or overestimate the observed ones. FA2 is the fraction of $\mathbf{C p}$ values (normalized to 1) within a factor two of corresponding $\mathbf{C o}$ values. The statistical index NMSE represents the model values dispersion in respect to data dispersion. The best results are expected to have values near zero for the indices NMSE, FB and FS, and near one in the indices COR and FA2. The analysis of the statistical evaluation shows a reasonable agreement between the computed values against the experimental ones.

\begin{tabular}{|c|c|c|c|c|c|c|}
\hline$\triangle t_{1}(s)$ & $\triangle t_{2}(s)$ & NMSE & COR & FA2 & FB & FS \\
\hline 600 & 60 & 0,36 & 0,75 & 0,67 & 0,21 & $-0,03$ \\
\hline
\end{tabular}

Table 1: Statistical evaluation of the puff model. 


\section{Conclusions}

Bearing in mind that the majority of puff models are based upon the Gaussian formulation for pollutant diffusion inside the puffs, in this work the authors are confident to affirm that the puff model was improved, in the sense that the pollutant dispersion is now described by the analytical GILTT solution of the three-dimensional diffusion equation, which is a proper solution either for homogeneous and non-homogeneous scenarios in atmosphere. The solution simplicity comes from the fact that the three-dimensional solution is written as a product of one-dimensional ones.

As preliminary evaluation of this model performance the predicted results encountered are compared against the Copenhagen data set, which has a greater time resolution in respect to the original one. The good statistical fitting of the results attained against experimental data, give us confidence to believe that the proposed model is a promising and particularly innovative attractive theoretical model to set up an operative computational code for pollutant dispersion in atmosphere. Our future attention shall be focus in this direction incorporating an improved analytical GILTT solution for eddy diffusivity also varying with time .

\section{Acknowledgements}

The authors thank CAPES, CNPq and FAPERGS for the partial financial support of this work.

\section{References}

[1] Chang, J.C and Hanna, S.R., Air quality model performance evaluation, Meteorol. Atmos. Phys., Vol. 87 (2004) 167-196.

[2] Gryning, S. and Holtslag, A. and Irwing, J. and Silversten, B., E., Applied dispersion modelling based on meteorological scaling parameters, Atmospheric Environment, Vol. 21 (1987) 79-89.

[3] Moreira, D. M., Vilhena, M. T., Buske, D. and Tirabassi, The State-of-art of the GILTT method to simulate pollutant dispersion in the atmosphere, Atmos. Research, Vol. 92 (2009) $1-17$.

[4] Moreira, D. M., Vilhena, M. T., Tirabassi, T., Costa, C. and Bodmann, B., Simulation of pollutant dispersion in atmosphere by the Laplace transform: the ADMM approach, Water, Air and Soil Pollution, Vol. 177 (2006a) 411-439.

[5] Pereira, L.P., Costa, C.P., Vilhena, T. and Tirabassi, T., Puff Models for Simulation of Fugitive Hazardous Emissions in Atmosphere, Journal of Environment Protection, Vol. 1 (2011) 154-161.

[6] Pleim, J. E. and Chang, J. S., A non-local closure model for vertical mixing in the convective boundary layer, Atmospheric Environment, Vol. 26A (1992) 965-981.

[7] Tirabassi, T. and Rizza, U., A practical model for the dispersion of skewed puffs, Journal of Applied Meteorology, vol. 34 (1995) 989-993.

[8] Tirabassi, T. and Rizza, U., Boundary layer parameterization for a non-Gaussian puff model, Journal of Applied Meteorology, vol. 36 (1997) 1031-1037.

[9] Van Ulden, A. P., A surface layer similarity model for the dispersion of a skewed passive puff near the ground, Atmospheric Environment, vol. 26 A (1992) 681-692. 